\title{
Language Accessibility in the Classroom: How UDL Can Promote Success for Linguistically Diverse Learners
}

\author{
Patricia Rice Doran \\ Towson University
}

\begin{abstract}
This article provides an overview of the Universal Design for Learning (UDL) framework, which is based on brain-structure research and which incorporates multiple means of instruction, action and expression, and engagement. The article describes the relevance of this framework to linguistically diverse and culturally and linguistically diverse (CLD) learners, and it provides a set of scenario-based examples of how UDL might help educators to implement effective instruction for a CLD learner in an inclusive setting. The paper makes additional suggestions for building on UDL principles to design linguistically accessible instruction for CLD learners.
\end{abstract}

Ms. Rivera, a seasoned fifth grade teacher at Elmwood Elementary, was asked to participate in a team meeting regarding her student Oscar, a culturally and linguistically diverse (CLD) student who also received special education services. Oscar's family had arrived in the country three years earlier, and Oscar had made friends at Elmwood but still struggled academically. Oscar struggled to complete daily assignments in all his content classes; in addition, he frequently seemed lost or confused as the class completed routine activities such as going to lunch, switching classes, or walking to an assembly. In working with Oscar and his mother, Ms. Rivera had learned that Oscar liked school and had strong family support, but found it challenging to complete all the required work and follow classroom instruction. This was consistent with what a multidisciplinary team had found the year prior in diagnosing Oscar with a mild learning disability. As the discussion continued, the team members found that each brought a specific expertise to the table for making modifications: Ms. Rivera knew the demands of the grade-level 
curriculum; Mr. Godwin could speak to Oscar's developing English proficiency; and Ms. Waters, the special educator, had suggestions about accommodations and learning strategies. The team spent half an hour brainstorming adaptations to an upcoming history unit, simplifying language, creating alternative assignments with reduced reading and writing demands, and creating a customized glossary so Oscar would better understand the historical and cultural references in the textbook. Halfway through, Ms. Rivera looked up and sighed. "I can't imagine doing this for every single lesson and unit. If only the curriculum were more accessible to begin with!"

This article addresses the relevance of the Universal Design for Learning (UDL) framework for students who are culturally and linguistically diverse (CLD), including those with and without disabilities. While the example of Oscar is fictional, there are, in reality, thousands of children like Oscar, who struggle to succeed in day-to-day classwork because instruction has not been designed with all learners in mind. For professionals such as Ms. Rivera and her colleagues, the process of making curriculum and language accessible is complex, requiring collaboration among general educators, teachers of English for Speakers of Other Languages (ESOL) or of other languages, bilingual education teachers, and special educators or educators with expertise in learning differences (Davison, 2006; Musti-Rao, Hawkins, \& Tan, 2011). In particular, ESOL or bilingual education staff contribute to this process with their knowledge of language acquisition, language structure, and linguistic strategies; while general educators may provide a deep familiarity with content. Special educators may bring to the process a more specialized understanding of learning and development and the appropriate teaching strategies and accommodations based on individualized education program (IEP) and special education goals. For students who are culturally and linguistically diverse and exceptional (CLD/E), as in the story above, collaborative input from all parties (including students and their families) is necessary to their success, but not sufficient; educators also need familiarity with current research on cognition and learning strategies to plan for and meet all students' needs. As a result, increasing numbers of support and intervention teams have begun to draw on the framework of UDL, a brain-based approach that emphasizes the use of multiple means of representing content, of providing to students varied means of action and expression, and of engaging students' interests (CAST, 2011; Gravel, Ralabate, \& Thomas, 2010). UDL is seen as one means of making instruction more accessible and appropriate for students with a range of needs, abilities, and learning styles, with its emphasis on flexible and challenging curriculum built for maximum accessibility.

\section{Background and History of UDL}

Initially an outgrowth of the assistive technology field, UDL encompasses a more holistic approach to education, focused on designing learning environments, curriculum, and instruction to ensure maximum accessibility, upfront, for varied groups of learners. The concept of UDL was pioneered in the mid-1990s by researchers at CAST, a Massachusetts centre that began with a focus on computer technology and children with disabilities. Over the years, CAST expanded its mission to address the broader question 
of how educators could teach in a way that allowed all learners to access the curriculum (CAST, 2014). Technology, still a key component of a UDL approach, was part of the answer, but this focus on technology was accompanied by an emphasis on brain-based learning and learner variability (National Center on UDL, 2012). Rather than focusing solely on accommodations that might occur during and after the lesson-planning process, UDL emphasizes instructional design focused from the very beginning on maximizing accessibility and minimizing barriers to learning while also engaging and challenging students. In 1998, the Council for Exceptional Children, the nation's leading professional organization for special educators, published a white paper on UDL, raising its profile among special educators (CAST, 2014). Over time, CAST developed and disseminated UDL principles, UDL guidelines (recommendations for educators to implement the UDL principles in the classroom), and checkpoints (concrete suggestions aligned to the three principles and to the guidelines; CAST, 2011). Numerous projects incorporating UDL into curriculum planning have been funded by the federal government and by professional organizations as well as school systems. The UDL-based resources available to teachers have expanded over the years, with UDL exemplar lessons, videos, and research articles available on CAST's website and on the website of the National Center on UDL (CAST, 2014).

\section{Characteristics of a UDL approach}

In a UDL-based approach, educators begin by identifying student strengths and needs, deciding upon the appropriate curriculum goal to target in each lesson or unit of instruction, and brainstorming potential barriers. Planning involves minimizing barriers and maximizing accessibility, with a corresponding emphasis on providing challenge for all learners, often through flexibility and the use of options (CAST, 2011). Instruction is aligned with three sets of brain networks identified through neuroscience: recognition, strategic, and emotional or affective (CAST, 2011). As a result, instructors meet the needs of all learners by including multiple means of representation (supporting recognition), multiple means of action and expression (supporting varied strategies), and multiple means of engagement (supporting affective networks). Educators whose practice is guided by UDL principles incorporate these ideas at all stages of the lesson, from preassessment through all stages of instruction, including assessment. Choice and flexibility are key elements of this approach, as providing options for students supports engagement and also helps teachers to differentiate activities to ensure content is presented in ways appropriate for various groups of learners. In this respect, UDL goes beyond differentiation or accommodations, which are often steps taken at a designated point in the lesson-planning process; rather, UDL is a "framework for understanding how to create curricula" designed to accommodate learner differences from the beginning (CAST, 2011, p. 4). The UDL principles (multiple means of representation, action and expression, and engagement) likewise support instruction for learners who bring many diverse needs, skills, and backgrounds to the classroom (CAST, 2011). 


\section{UDL and Diverse Learners}

As classrooms become more diverse, with growing numbers of students who are CLD (Aud et al., 2012), it is increasingly timely to think about the ways in which a UDL-based approach can help us to support students who have specific language needs, including those who are English learners as well as those with exceptionalities. When professionals think about supporting learners who are CLD, including those with and without disabilities, they often begin by planning curriculum modifications and accommodations for each diverse learner. However, UDL is built on the principle that good instruction for these students goes beyond accommodations or modifications; it starts with ensuring maximum accessibility in all areas of instruction and assessment, which may ultimately render some accommodations unnecessary. (For example, if every student has a laptop or tablet, then a keyboard accommodation for a student with a writing disability is no longer necessary - his or her needs are met already.) For CLD learners, accessibility must be conceptualized broadly, in cultural and affective ways as well as task-delimited ones; students approaching a textbook reading on the SpanishAmerican War may each need different amounts of historical or cultural context, or may view the subject differently based on their national background or personal experiences with conflict. Additionally, varied approaches for procuring students' investment in the content, and emotional response to the learning experience, can support student learning by fostering increased engagement and providing a safe, welcoming space within the classroom.

Through providing multiple means of content representation, varied options for response, and multiple means of learner engagement (Grapinski, Gravel, \& Rose, 2010), UDL attempts to build on students' diverse strengths as well as their challenges, linguistic and otherwise. For CLD learners, their language and cultural diversity is in fact a strength even when school systems and curricula may regard it as a deficit to be remedied instead of an asset (Fiedler et al., 2008; Gay, 2010). One advantage of using UDL for this population is that UDL does not dictate what to teach, or even how to teach it - decisions that are rooted in curriculum guidelines and in awareness of individual students' strengths, backgrounds and needs. Rather, UDL provides a roadmap for educators to think through the process of identifying barriers to learning and working to remove them. This process is relevant for all learners, whatever their subject, grade level, or language and cultural background. In fact, UDL must be implemented within the context of a student's unique culture, not outside it or as an attempt to supersede it. The use of options, choice, and flexibility, key to a UDL framework, is one potential way to address the needs of all students in a culturally responsive manner that increases engagement and learner empowerment (CAST, 2011; Collier, 2010; Enright, 2011; Marinak \& Gambrell, 2008). Options and flexibility also provide room for educators to differentiate lesson content for cultural relevance, varied learning styles, and diverse language needs within the same classroom.

Another strength of UDL is its implicit reliance on equity. Traditionally, educators have designed instruction for a homogeneous and typical mainstream or "average student" (National Center on UDL, 2012) and then retrofitted for CLD learners, those with disabilities, and those who learn in different ways. In prioritizing the needs of all students equally and considering the needs of diverse learners from the first moment of 
planning, UDL offers a powerful tool for equitable and responsive instruction (Mason \& Webber, 2003).

The idea of UDL itself is situated within Western medical and biologically based models, with its emphasis on brain structure and function (CAST, 2011; Rose, Meyer, \& Hitchcock, 2005); however, the UDL framework (CAST, 2011; CAST, 2014) can be tremendously beneficial for teachers seeking practical ways to accomplish coexisting goals of accessibility, challenge, and cultural appropriateness, an increasing area of focus in education (Fiedler et al., 2008; Gay, 2010). Chita-Tegmark, Gravel, Serpa, Domings, and Rose (2012) have provided an overview of how the UDL framework can be used to support students from diverse cultural backgrounds, drawing on what is known about interfaces between brain-based differences and culture. They identify particular applications of the UDL guidelines, such as focusing on overarching ideas and providing repeated opportunities for practice, which can support students from varied backgrounds and also accommodate differences in learning modalities that may result from cultural, as well as other, factors (Chita-Tegmark et al., 2012). UDL is also a powerful way to conceptualize our instruction for those students who are linguistically diverse, who also have distinct learning needs (Chita-Tegmark et al., 2012). These students stand to benefit from an approach that sees their bilingualism or multi-lingualism as a strength to be supported through instruction, not a deficit to be remedied or removed through elimination of the native language from the classroom. Deployed within a multicultural framework, UDL can remind teachers that multiple avenues and means of expressionincluding linguistic supports - can supplement and enrich the dominant classroom language and culture.

\section{Language as an Element of UDL}

Through its emphasis on the language of instruction, the UDL framework provides opportunity for teachers to think about the accessible classroom not just in terms of physical layout but also in terms of language accessibility. As the medium through which the majority of instruction is delivered, language plays several roles in the classroom. Accessible language can support students' growth and learning, enhance their developing language proficiency (in both English and their native language), and support students' social and emotional integration into the school and community. Language theorists draw distinctions between social or playground and cognitive or academic language skills (Cummins, 2003). However, the reality is that many classroom interchanges, particularly those in which students have opportunities for rich use of authentic oral language, mix academic and social demands and therefore require skill in both social and academic English. For that reason, special educators, as well as their general education and ESOL colleagues, must build in language accessibility as a foundational element of all instructional decisions. This means providing opportunities for language practice and student choices regarding language wherever feasible. Such opportunities are supported through recommendations in the UDL guidelines, which explicitly address language and communication (such as Guideline 2, "Provide options for language, mathematical expressions, and symbols"), providing "checkpoints" (e.g., Checkpoint 2.1, "Clarify vocabulary and symbols") and classroom-based examples to help educators apply the guidelines to their own classrooms. The example below, building on the fictional scenario 
presented at the beginning of the article, describes how Oscar's teachers worked within a UDL framework to ensure instruction was linguistically accessible and included appropriate supports.

Ms. Rivera and her team met again the following week to continue planning for Oscar. Ms. Waters, the special educator, had brought some information on UDL that she had received at a prior workshop. The team decided to focus on UDL principles in making sure instruction was accessible to Oscar, starting with science, a course he was currently failing. Oscar seemed to struggle to follow directions in hands-on activities, and he was also struggling to keep up with challenging vocabulary work and lengthy reading and response assignments. After some brainstorming, the team came up with some immediate strategies for whole-class instruction, none of which would take Ms. Rivera a long time to implement each day but which would help Oscar as well as other learners. First, focusing on multiple means of representation, the team decided to provide Oscar with native-language materials and to help Ms. Rivera incorporate visuals (including pictures and videos) into each activity. Ms. Rivera set a goal of having three different ways of presenting any key information, combining direct spoken instruction, visual notes, drawings and diagrams, videos, and informational text readings. Ms. Rivera also would review directions for every activity and make sure she modelled, provided visual directions, and gave oral directions for each activity. Next, the team focused on incorporating multiple means of action and expression. They developed a tic-tac-toe menu that listed different activities students could select for homework, suitable for use each week. The ESOL teacher offered to place a Spanish-language version of the menu on the back of the English one so that Oscar would have clarity on homework directions. Second, they determined that Ms. Rivera would use pair-sharing discussion at least twice in each lesson, matching Oscar with peers who spoke his native language so that he could choose to communicate in either language. The ESOL teacher also gave Ms. Rivera several graphic organizers, which Oscar had used in his ESOL classes; she could make these available to the entire class as optional writing supports. Last, the team considered using multiple methods to engage Oscar (and others) in science. By the end of the meeting, Ms. Rivera had a list of high-interest activities and openers, such as film clips related to science and connections to household objects and real-life events. The ESOL teacher even lent her a book of jokes to incorporate into lessons, which helped to support Oscar's developing knowledge of English idioms while keeping his classmates engaged and entertained. As one of the most important ways to support Oscar, the teachers discussed these proposed changes with Oscar and his family via a quick conference before school, using an interpreter and asking Oscar's parents to let them know if the changes seemed to be helping him understand the content better at home. As Ms. Rivera began to implement the new strategies, she found with surprise that all her students-not just Oscar-demonstrated greater engagement in class and higher rates of homework completion using the new tic-tac-toe board.

\section{Designing a Language-Accessible, UDL-Based Classroom}

Basic principles of UDL, such as providing visuals and other means of representing content, or offering choices for activities and work products, serve learners who are CLD and CLD/E particularly well. In Oscar's case, his teachers collaboratively identified ways 
to make instruction more accessible and allow him to accomplish rigorous and challenging tasks. The multidisciplinary expertise of this team, with representation from ESOL, general education, and special education, allowed educators to efficiently eliminate several language barriers and provide options to improve Oscar's comprehension and retention of content. However, when not all team members bring language-related expertise to the table, it can be challenging to determine where to start or how to streamline language while maintaining rigour. In addition to the general UDL principles, guidelines, and checkpoints (CAST, 2011), , some helpful practices for CLD learners are useful as well.

\section{Five Best Practices}

These suggestions, drawn from practical experience and the literature, are not identical to the UDL guidelines; and the author's intention is not to present these as an explicit part of the UDL framework. However, in many cases these best practices for CLD learners are related to or consistent with UDL guidelines as illustrated by the checkpoints listed below.

1. Build in multiple and varied opportunities for oral language. See Figure 1 for a list of strategies for implementing this practice.

- Consistent with UDL Checkpoint 2.4, "Promote understanding across languages" (CAST, 2011).

Figure 1: Oral Language Strategies

\section{Six Strategies for Incorporating Oral Language into Daily Instruction}

- Pair-share / partner work

- Oral summary and paraphrase of content, student responses, directions

- Literature circles or similar reading activities completed with peers

- Use of "conversation starters"

- Reciprocal teaching

- Purposeful teaching of question / answer stems, academic discourse

2. Provide explicit instruction, synonyms, and native language support for key words - not just key technical vocabulary, but directions, unfamiliar terms, and even low-context words such as prepositions (Beck, McKeown \& Kucan, 2005).

- Consistent with UDL Checkpoint 2.1, "Clarify vocabulary and symbols"; UDL Checkpoint 2.2, "Clarify syntax and structures" (CAST, 2011). 
3. Use consistent vocabulary and syntax (grammar) structures for beginning English learners. As students' language proficiency increases, teachers can likewise increase the complexity and variety of their language, although using consistency as they introduce academic language to the classroom will continue to be helpful (Fisher, Frey, \& Rothenberg, 2008).

- Consistent with UDL Checkpoint 2.2, "Clarify syntax and structures" (CAST, 2011).

4. Encourage self-assessment and metacognitive thinking, in a student's native language as well as in English (Zwiers, 2008).

- Consistent with UDL Checkpoint 6.4, "Enhance capacity for monitoring progress" (CAST, 2011).

5. Design lessons to support mastery learning, offering all students opportunities to repeat and practise until a skill is consistently demonstrated. As CLD learners have a wide range of background knowledge, experience, and skills, they benefit from repeated opportunities for practice and extension of knowledge.

- Consistent with UDL Checkpoint 3.3, "Guide information processing, visualization, and manipulation"; UDL Checkpoint 3.4, "Maximize opportunities for transfer and generalization" (CAST, 2011).

\section{Strategies Specific to Academic Language}

Educators should also be aware of challenges specific to technical or academic language, particularly problematic for CLD learners (Cummins, 2003). Academic language, more likely to be abstract and challenging and less likely to be linked in familiar context or everyday activities, can be especially difficult for students with language needs - both those who have language-based exceptionalities and those who are CLD. The following strategies can improve language accessibility in academic text and discourse; the example farther below extends the scenario with Oscar's teachers to demonstrate the positive impact of supports for academic language and higher-level thinking.

1. Rephrase using familiar vocabulary. Particularly for students whose vocabulary is still developing, teachers can build language skills by providing an "oral gloss," using a familiar synonym immediately after using an unfamiliar vocabulary word. For example, a teacher might elaborate on the word "precipitation," when it occurs in a sentence by saying something like the following: "The text said a drought can occur when there is not enough precipitation, or rain and snow." Another option is to assist students by providing native-language support; where teachers lack dual language proficiency, tools such as bilingual dictionaries or online translators can be paired with extensive use of pictures to teach vocabulary.

- For further discussion of this concept in a UDL context, see Checkpoint 2.1, "Clarify vocabulary and symbols" (CAST, 2011).

2. Simplify syntax. For example, directions are often needlessly complex. "After we eat lunch, if it is not raining, I would like everyone to come in the way we usually do, get 
your books if you do not have them already, and take your seat on the carpet" can easily be reduced to, "After lunch, please go to the carpet. Bring your books." This strategy can be used to simplify written text as well as spoken language. When teachers do not have time to rewrite text, students can work in groups or pairs to provide simplified summaries or paraphrasing, which can support learners who are CLD as study materials.

- For further discussion, see UDL Checkpoint 2.2, "Clarify syntax and structure" (CAST, 2011).

3. Build in options for response that do not require extensive language production but which encourage critical thinking (in a student's native or second language). Sorting, ranking, listing, and drawing are excellent activities (Collier, 2010). Each of these provides opportunity for higher-level thinking skills without requiring language-heavy responses.

- UDL Checkpoint 7.1, “Optimize individual choice and autonomy (CAST, 2011).

4. Guide students through text using key questions and skeletal outlines whose structure mirrors that of the reading. A main idea question ("What three factors influence global trade?") and bulleted list of the three factors influencing global trade might be paired with a social studies selection that describes each factor.

- UDL Checkpoint 3.3, "Guide information processing, visualization, and manipulation"; UDL Checkpoint 5.3, "Build fluencies with graduated levels of support for practice and performance" (CAST, 2011).

5. Visually simplify complex material. Teachers can condense text or use a highlighter or pencil to mark key sections of text. Initially, a special educator, ESOL or classroom teacher, or even reading specialist might model how to do this; as the student becomes more familiar with the process, he or she might take more responsibility for identifying key sections of the text.

- UDL Checkpoint 2.2, "Clarify syntax and structure"; UDL Checkpoint 2.3, "Support decoding of text, mathematical notation, and symbols" (CAST, 2011).

As the semester continued, Ms. Rivera and her team became excited about the way that they restructured the fifth grade's activities to support language and content learning for Oscar. For example, his teachers had begun using consistent directions and formatting on papers across all subject areas. After every activity or assessment, the class would stop to debrief and talk about what strategies they had used, with Oscar being given a bilingual reference sheet for some of the strategies and metacognitive words. Additionally, the ESOL teacher had created a picture reference chart for prepositions and content vocabulary for Oscar to keep in his folder, but it was so popular that she had ended up giving one to each student in the class. Finally, the use of more small-group activities, combined with more emphasis on metacognition and student selfmonitoring, had created a classroom climate where teachers had more freedom to circulate and support the students like Oscar who needed extra help. The team members noticed that all students seemed to benefit from these changes, and test scores rose while student behaviour improved. As the spring continued, with her team's encouragement, 
Ms. Rivera allowed Oscar to discuss his ideas before writing them down and, subsequently, encouraged him to write a little bit more each time. Seeing his positive responses to this approach, she had the idea to implement the strategy with all her learners. As the team continued to plan, their discussions gradually began to focus on making the content accessible, engaging, and challenging-for Oscar and all of his peers.

Meanwhile, the original goal of improving Oscar's achievement also seemed to be met. Oscar had begun to participate in, and even initiate, discussions and class activities. While written language was still an area of challenge for him, his increased understanding of classroom activities and instruction, coupled with extra time to process ideas through partner discussion, was slowly helping to improve the quality of his own writing.

\section{Gradually Add Challenge}

A final element of UDL, sometimes neglected as educators focus on accessibility and support, is the importance of rigour and challenge (CAST, 2011; Rose, Meyer, \& Hitchcock, 2006). UDL is not meant to reduce expectations to an artificially low level or to encourage instruction at the lowest common baseline, but rather to improve accessibility and to provide embedded supports for all, which can be faded as students gain skills and confidence. Instruction should always be a balance between challenge and support. For learners who are CLD, this idea parallels the concept of comprehensible input, the idea that instruction should take place at or slightly above the student's language level in order to facilitate continued growth as well as understanding (Krashen, 2003; Vygotsky, 1978). In practice, this principle requires flexibility on the part of teachers. For example, one might ensure access to key vocabulary in a lesson by using one consistent term at the beginning of the lesson, gradually and explicitly introducing related synonyms throughout the lesson in order to expand students' vocabulary by building on existing knowledge. In the fictional example, Oscar's teachers drew on this principle in gradually increasing writing expectations, giving him additional challenges once they knew he was prepared and able to meet them.

\section{Conclusion}

As illustrated by the story of Oscar, supporting learners who are CLD or CLD/E is a long-term endeavour. This endeavour requires collaboration among multiple professionals as well as families, a proactive and problem-solving mindset, and flexibility. In the scenario involving Oscar and his teachers, these principles are illustrated by his teachers' focus on understanding of daily classroom routines; efforts to teach vocabulary; and increased academic expectations as the year continued. Teachers dealing with similar, real-life situations may do well to incorporate a similar assessment of students' language needs and willingness to make adaptations where needed. As illustrated in this story, such efforts can not only improve performance of individual CLD students but, also, create a more accessible classroom environment for all learners. An emphasis on language accessibility, combined with the desire to add support when necessary and challenge when feasible, will allow students to meet the high expectations appropriate for all learners. 


\section{References}

Aud, S., Hussar, W., Johnson, F., Kena, G., Roth, E., Manning, E., Wang, X., \& Zhang, J. (2012). The condition of education 2012 (NCES 2012-045). Washington, DC: U.S. Department of Education, National Center for Education Statistics. Retrieved from http://nces.ed.gov/pubsearch

Beck, I. L., McKeown, M. G., \& Kucan, L. (2005). Choosing words to teach. In E. H. Hiebert \& M. L. Kamil (Eds.), Teaching and learning: Bringing research to practice (pp. 207-242). Mahwah, NJ: Lawrence Erlbaum.

CAST. (2011). Universal Design for Learning guidelines version 2.0. Retrieved from http://www.udlcenter.org/aboutudl/udlguidelines

CAST. (2014). CAST through the years: One mission, many innovations. Retrieved from http://www.cast.org/about/timeline/index.html

Chita-Tegmark, M., Gravel, J., Serpa, M.B., Domings, Y., \& Rose, D.H. (2012). Using the Universal Design for Learning framework to support culturally diverse learners. Journal of Education, 192(1), 17-22.

Collier, C. (2010). Tier 1 interventions. In RTI for diverse learners: More than 200 instructional interventions. pp. 13-68. Thousand Oaks, CA: Corwin.

Cummins, J. (2003). Reading and the bilingual student: Fact and friction. In G. G. Garcia (Ed.), English learners: Reaching the highest level of English literacy (pp. 2-33). Newark, DE: International Reading Association.

Davison, C. (2006). Collaboration between ESL and content teachers: How do we know when we are doing it right? International Journal of Bilingual Education \& Bilingualism, 9(4), 454-475.

Enright, K. A. (2011). Language and literacy for a new mainstream. American Educational Research Journal, 18(1), 80-118.

Fiedler, C., Chiang, B., Van Haren, B., Jorgensen, J., Halberg, S., \& Boreson, L. (2008). Culturally responsive practices in schools. Teaching Exceptional Children, 40(5), 52-59.

Fisher, D., Frey, N., \& Rothenberg, C. (2008). Appendix: List of instructional routines. In Content-area conversations: How to plan discussion-based lessons for diverse language learners. Alexandria, VA: ASCD. Online book retrieved from www.ascd.org/publications/books/108035.aspx

Gay, G. (2010). The pedagogical potential of cultural responsiveness. In Culturally responsive teaching: Theory, research and practice. New York, NY: Teachers' College Press.

Grapinski, S. L., Gravel, J. W., \& Rose, D. H. (2012). An introduction to UDL: Questions and answers. In T. Hall, A. Meyer, \& D. Rose (Eds.), UDL in the classroom: Practical applications (pp. 1-8). New York, NY: Guilford.

Gravel, J., Ralabate, P., \& Thomas, L. (2010). UDL: A framework for access and equity. Leadership for Excellence and Equity Forum 2010: Phoenix, AZ. Retrieved from http://www.slideshare.net/NCUDL/udl-a-framework-for-access-and-equity

Krashen, S. (2003). Explorations in language acquisition and use: The Taipei lectures. Portsmouth, NH: Heinemann.

Marinak, B., \& Gambrell, L. (2008). Intrinsic motivation and rewards: What sustains young children's engagement with text? Literacy Research and Instruction, 47, 9-26.

Mason, C., \& Webber, J. (2003). And learning for all. Principal Leadership, (April 2003), 30-33.

Musti-Rao, S., Hawkins, R., \& Tan, C. (2011). A practitioner's guide to consultation and problem solving in inclusive settings. Teaching Exceptional Children 44(1), 28-36. 
National Center on Universal Design for Learning. (2011). Frequently asked questions. Retrieved from http://www.udlcenter.org/advocacy/faq_guides/ell\#question2)

National Center on Universal Design for Learning. (2012). Learner variability and UDL [Online seminar presentation]. UDL Series, No. 1. Retrieved from http://udlseries.udlcenter.org /presentations/learner_variability.html?plist=explore

National Center on Universal Design for Learning. (2013). UDL Resource Library. Retrieved from http://www.udlcenter.org/resource_library

Rose, D. H., Meyer, A., \& Hitchcock, C. (2005). Introduction. In D. H. Rose, A. Meyer, \& C. Hitchcock (Eds.), The universally designed classroom: Accessible curriculum and digital technologies (pp. 1-12). Cambridge, MA: Harvard University Press.

Vygotsky, L. S. (1978). Mind in society. Cambridge, MA: Harvard University Press.

Zwiers, J. (2008). Developing the language of thinking. Educational Leadership, 65(9). Retrieved from http://www.ascd.org/publications/educational-leadership/archived-issues.aspx

\section{Author's Note}

Correspondence concerning this article should be addressed to Patricia Rice Doran, Department of Special Education, Towson University, 8000 York Rd., Towson, MD 21252, USA. Email: pricedoran@towson.edu 